Preface

\section{Respiratory Viral Infections}

\author{
Sunit K. Singh, $\mathrm{PhD}^{1}$ \\ ${ }^{1}$ Molecular Biology Unit, Institute of Medical Sciences (IMS), Banaras
}

Hindu University, Varanasi, India

Semin Respir Crit Care Med 2016;37:485-486.

Viral infections represent a major cause of respiratory tract infections globally. The incidences of viral infections are very high among infants and old age groups. The respiratory viral infections often may lead to complications such as acute otitis media and asthma exacerbations. The respiratory viruses may add an additional layer of complexity for the children suffering from asthma, which may lead to the complications such as pneumonia. Neonates with weak immune systems may be at higher risk for respiratory complications. The clinical presentations of various respiratory viral infections overlap with each other. Therefore, the precise diagnostic tools of respiratory virus infections help in patient management and prevention of secondary spread. The development of novel molecular detection methods as well as better understanding of the pathogenesis of respiratory viruses is required to handle the potential outbreaks.

Viral infections are common causes of respiratory tract disease in the outpatient settings of the hospitals without the isolation units. Some viruses, such as influenza, respiratory syncytial virus, metapneumovirus, as well as parainfluenza virus, and coronavirus infections are relatively common. It is very important to recognize these viral infections for the treatment, infection control, and public health measures. An understanding of the viral pathogenesis, along with host response, is required for the patient care and public health response. The host responses to these infections may vary from person to person. All can cause respiratory disease but a few are of great public health concern, particularly

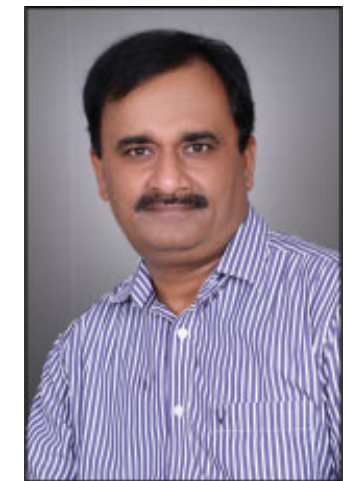

Sunit K. Singh, PhD novel strains of influenza, adenovirus, severe acute respiratory syndrome (SARS), and Middle East respiratory syndrome (MERS). The close association of the animals and human hosts results into the transmission of respiratory viral infections to the human population. The most common spill overs from the animals to the human population include the outbreaks of avian influenza, SARS-CoV infections, and MERS-CoV infections. The identification of the novel viruses circulating in the human population requires more sensitive newer technology platforms. In addition, the "one health" concept is necessary to handle the outbreaks of the respiratory viral infections.

I have greatly enjoyed my interaction with the large panel of renowned virologists and biomedical scientists, who have contributed their articles in this special issue. I am highly thankful to Prof. Joseph P. Lynch III, Editor-in-Chief of the journal Seminars in Respiratory and Critical Care Medicine, for his help and support in publishing this special issue on the "respiratory viral infections."
Address for correspondence Sunit K. Singh, PhD, Molecular Biology Unit, Laboratory of Human Molecular Virology and Immunology, Faculty of Medicine, Institute of Medical Sciences (IMS), Banaras Hindu University (BHU), Varanasi 221005, India (e-mail: sunitsingh2000@gmail. com).
Issue Theme Respiratory Viral Infections; Guest Editor: Sunit K. Singh, PhD
Copyright (๑) 2016 by Thieme Medical Publishers, Inc., 333 Seventh Avenue, New York, NY 10001, USA. Tel: +1(212) 584-4662.
DOI http://dx.doi.org/ 10.1055/s-0036-1584802. ISSN 1069-3424. 